Aller: Is it possible to use a focal plane spectrograph at a large telescope with a greater focal ratio to alleviate effects of overlap of spectra of stars?

Courtès: The question of Professor Aller permits me to develop my remark about the limit in using any objective-prism techniques.

An objective prism has a focal length always limited by the seeing condition, as 1 " of seeing corresponds to $25 \mu$ for $5 \mathrm{~m}$ focal length, so you see that it is difficult to use focal length longer than $5 \mathrm{~m}$ and $I$ see the certain limit around $10 \mathrm{~m}$. When you want to use a large telescope, for example $2 \mathrm{~m}$ aperture, the focal ratio is of course too high, $2 \cdot 5$ or, at the limit, 5 , or $10 \mathrm{~m}$ equivalent focal length. That gives you a new limit in reaching faint stars because of the sky fog rising fast in relation to the focal ratio. In spite of that, $I$ have designed an adaptation of the Fehrenbach prism to the Reducteur focal usually used for nebular work - but this instrument will be equipped with filters selecting some of the most characteristic parts of the spectra. Focal ratio 3 will be used; this corresponds to approximately $6 \mathrm{~m}$ focal length.

Kerr: Professor Fehrenbach has published two examples of the frequency distribution of velocities in particular areas. These are directly comparable with 21 -cm line profiles. Is he planning to extend this form of presentation?

Courtès: Certainly, but that will be made at the end of this general survey.

Eggen: We have heard of possible connections between the two Clouds and between Clouds and the Galaxy. Is a search for these connections proposed?

Courtès: Some stars have an intermediate radial velocity and could be interpreted as intermediate distance stars - but this selection will be easier for Clouds-to-Galaxy than for Cloud-to-Cloud.

\title{
52. MEMBER STARS OF THE MAGELLANIC CLOUDS FROM PROPER MOTIONS
}

\author{
SIR RichaRd WoOLLEY \\ Royal Greenwich Observatory
}

The material which I wish to present today is set out in Royal Observatory Bulletin Number 66, of which I have a few advance copies with me. The paper describes measures of about 700 stars in an area about $1^{\circ}$ square. These are nearly all the stars down to a limit of $V=14 \mathrm{~m} 5$ in the area. Proper motions were measured by comparison between two plates taken on 1912 Jan. 21 and 1960 Nov. 16-17. The earlier plate had an exposure of $5 \mathrm{hr}$, which is exceptionally long for astrographic plates of those times. The measures of $\Delta x$ and $\Delta y$ (the shifts in units of onetenth of a micron in the directions of right ascension and declination) were reduced by constructing plate constants such as would make the least-squares motion of the stars with $B-V<0$ zero. In the reduction 104 such stars were used. The standard errors of measurement are $\sigma(x)= \pm 15 \cdot 9$ and $\sigma(y)= \pm 14 \cdot 9$, assuming that these stars really are members of the LMC and exhibit no relative proper motion. By using these plate constants, values of $\Delta x$ and $\Delta y$ were computed for all the remaining stars. The stars with $0.5<B-V<1.5$ (apart from cepheids and a few reddened stars) are very plainly shown by their proper motions to be nearly all field stars, and indeed their motions can be satisfactorily explained by supposing them to be galactic stars showing the parallactic motion, galactic rotation, and a velocity ellipse appropriate to galactic stars. Stars with $B-V>1.5$ cannot be determined to be members of the LMC on proper motion alone, but other considerations suggest 
that this is so. For example, their $x, y$ distribution in the field follows the same pattern as that shown by the very blue stars, whereas the field stars $(0 \cdot 5<B-V$ $<1 \cdot 5)$ are uniformly distributed over the plate.

Many persons on the staff of the Royal Greenwich Observatory took part in the work. I am particularly indebted to H. M. Astronomer at the Cape, Dr. R. H. Stoy, for the magnificent cooperation which he has set up between his Observatory and Greenwich, and to Dr. A. D. Thackeray, the Radcliffe Observer, for help in many directions.

\title{
Discussion
}

Tifft: How large an area in the Clouds can be eventually studied in this manner?

Woolley : Long exposure plates were taken with the Cape astrograph which cover all of the LMC; some of these are not of such good quality as the one we have used. We certainly plan to continue the work but we feel it necessary to determine $B$ and $V$ for all the stars whose proper motion we measure, in order to be able to interpret the results, and we have now asked Dr. Stoy to organize the setting up of suitable photoelectric sequences.

Arp : Are there any suitable first epoch plates available for the SMC?

Woolley: Unfortunately, no.

Bok: Would you please consider using old plates now at Harvard for measurement of proper motions? The old Bruce plates and the Metcalf plates should yield annual proper motions to within \pm 0 ". 010 - possibly considerably better. These old plates together with new ones can be used effectively now for the elimination of foreground stars.

Woolley: Dr. Menzel has agreed in principle to lend us these plates and I hope to arrange for one of the senior members of the Greenwich staff to go and inspect them.

Arp: Dr. Woolley reports that in the $V=13$ to 14 mag range in the LMC there is an absence of members with $B-V$ from 0.6 to $1 \cdot 5$. This agrees exactly with the colour-magnitude diagram of NGC 330 in the SMC. I would expect, however, that as one penetrates to the $V=16$ mag range the majority of evolved giants would be precisely in this colour range.

Woolley: I hope to present evidence on Thursday which shows that the stars down to $V=16^{\mathrm{m}}$. 5 in the colour range $0.5<(B-V)<1.5$ are still not members of the LMC.

Gascoigne: Both Melbourne and Yale-Columbia should have suitable first epoch plates of the Clouds. Melbourne in particular have some astrograph plates of the Large Cloud taken last century. Perhaps Mr. Harley Wood knows something about these.

Wood: The Melbourne chart plates have been at Greenwich. They do not reach magnitudes as faint as those discussed by the Astronomer Royal.

\section{THE SURFACE DISTRIBUTION`OF CLUSTERS, PLANETARY NEBULAE, SUPERGIANT M STARS, AND CARBON STARS IN THE LARGE MAGELLANIC CLOUD}

\author{
B. E. WesterLund \\ Mount Stromlo Observatory
}

\section{The Clusters}

Some years ago the plates used for the extended Uppsala-Mount Stromlo Atlas of the Magellanic Clouds were searched for clusters in order to compare the optical extension of the Large Cloud with the one defined by the radio-astronomical 